\title{
Review
}

\section{Looking Back on the 40 Years of English Journal Publication in Lighting}

\author{
Takashi IRIKURA
}

Board Member of English Journal Committee

Shibaura Institute of Technology

This year marks the 40th anniversary of the Journal of Light \& Visual Environment (JLVE), the IEIJ's English language publication. When the JLVE was first launched, only few people might have thought that it would continue for such a long time. Similar attempts were made by other institutes, but many of which resulted in ceasing their publication. There were strong pressure raised several times to quit the JLVE also in the IEIJ, mainly because of the financial difficulties. Thanks to the efforts by the chairman of the JLVE at the time and other people, it survived and has continued to this day.

The reason for the existence of the IEIJ in the first place is to provide opportunities to report the results of research and to exchange the information about the latest technologies. In particular, it is important to announce the results of research in English to the world, which is achieved by holding international conferences and publishing the journal in English. Discontinuation of the JLVE, even by financial reasons, would decrease the value of the Institute by half, which seems not yet well understood.

For the first 10 plus years, the JLVE was issued biannually. In the initial period, because of the difficulty in collecting enough number of papers, editorial managers were obliged to take turns to write papers and papers that had already been published in Japanese were slightly modified and translated into English for publication, which invited frowns. For the purpose of making up for the insufficiency of the number of contributions, papers that won the Excellent Paper Award or Research Encouragement Award are translated into English and included in the journal with a notice that they are translations, which has continued up to now.

Subsequently, through the efforts such as encouraging presenters in international conferences to contribute their papers and waiving submission fees, the number of papers contributed gradually increased. In particular, there were initially few contributions from overseas, but now many of the contributions are from oversees. Unfortunately, though, there has been some decline in the quality of papers along with the increase of the number of submissions and the number of rejected papers has increased as well.

We started using the J-STAGE site a few years ago for submission of papers and referee reviews on the Web. It is believed to have shortened the review time but far from sufficiently. It may be partly because of unfamiliarity but one reason is the complexity of usage.

Recently, we use the J-STAGE site for people to view and download papers already published and for publishing papers more quickly. Printed publication is carried out once a year for reduced costs as well as improved convenience. To take the opportunity of the 40th anniversary of the launch and 100th anniversary of the foundation of the IEIJ, we have decided to rename the English title of publication the Journal of Science and Technology in Lighting. The cover design has also been changed. One reason for the title change is that the former name, the Journal of Light \& Visual Environment, leads a misunderstanding of the researchers in the field of the "hardware category" such as light sources, circuits and physics based matters that this journal is limited to the field of "software category" because of the words "environment", although the journal is covering all the fields related to light and lighting. In order to solve this misunderstanding and to attract the submissions from the field of "hardware category" we decided to change the name of the journal to the new name "the Journal of Science \& Technology in Lighting", which clearly shows that the journal is covering all the field of lighting including "hardware category" as well as "software category".

Up to now, in respect of technologies relating to the development of illumination, Japan has always been a world leader. Of the six great inventions concerning incandescent lamps, two have been achieved by Japanese. In addition, energy conservation has been progressing around the world thanks to the popularization of LED lighting and advances in technologies. This is a benefit brought by the invention and development of blue LEDs by Japanese. 
In respect of the development of lighting technologies and research on light environment, Japan is still leading the world. When it comes to spread information to the world, however, Japan is lagging behind. One reason is the high hurdle of the English language. While globalization is now called for, publication of papers in English has still a long way to go. Technically high level papers in English guaranteed by reliable referee review are read by many people around the world. I would really like to encourage paper publications in English.

What is required of the JLVE is papers written in good English, not to mention publication of high-quality papers. For the author whose native language is not English the most difficult challenge is translation from his/her native language into English. A paper properly written with the basic structure of the language observed should lead to a relatively correct translation. However, if sentences not composed in a proper manner are translated as they are, the result would not make sense. Therefore, before translation, writing papers in correct native language is important.

Lastly, I would like to express my sincere gratitude to former chairmen of the JLVE Messrs. Satoru Kawai and Koichi Ikeda, who have contributed to its continuation and development since its launch. For writing this article, I have made a quotation from "In Greeting the 30th Anniversary of "The Journal of Light \& Visual Environment", written for the Japanese language journal by Mr. Satoru Kawai. 